\title{
Eradication success for non-tuberculous mycobacteria in children with cystic fibrosis
}

\author{
To the Editor:
}

Non-tuberculous mycobacteria (NTM) are an emerging pathogen worldwide in both cystic fibrosis (CF) and non-CF pulmonary disease (PD), with reports suggesting an increasing prevalence $[1,2]$. It is an opportunistic infection acquired from the environment [3], though conflicting evidence remains around person-to-person transmission [4, 5]. Recent evidence suggests that Mycobacterium abscessus complex (MABSC) may be the most detrimental airway infection to lung function in CF [6], yet its treatment remains poorly evidenced.

The paediatric CF service at the Royal Brompton Hospital (RBH) has had standardised diagnostic and treatment guidelines since 2011, which follow the American Thoracic Society (ATS) guidelines for the diagnosis of NTM-PD [7] and latterly CF-specific consensus guidelines [8, 9]. In CF, the two most commonly encountered species are MABSC and Mycobacterium avium complex (MAC), with the former more prevalent in the UK and the latter in the USA $[1,10]$. A recent consensus statement on treatment outcome definitions could not reach consensus on a definition of cure, but a microbiological cure was agreed as the end of anti-microbial treatment after culture conversion [11].

There are no published paediatric or adult CF randomised controlled trials of NTM treatment. Additionally, there are no established estimates of eradication success for NTM-PD in children, while there are limited reports from mixed adult/paediatric cohorts $[12,13]$. This cohort study aimed to establish NTM prevalence and treatment success in children with CF, and to identify any factors that may affect treatment success.

Electronic records for all children at the RBH CF centre were reviewed to identify cases with at least one new respiratory NTM infection between 2011 and 2018. Samples for NTM were taken at annual review, opportunistically if productive of sputum and from bronchoalveolar lavage (BAL), then grown in liquid culture medium, speciated with the Hain NTM-DR Test, inducible macrolide resistance detected by the Hain assay and VNTR typing performed by a national reference laboratory. Demographic data, spirometry, treatment details and associated diagnoses were taken from the visit closest to the first NTM growth. Treatment decisions were based on local guidelines [14], also taking into account antimicrobial susceptibility testing. Airway samples were tested every 3 months throughout treatment and for 12 months following treatment completion. The time points captured were time to start of treatment from first growth, time to culture conversion, length of treatment, and successful eradication. Transient infection is any isolate that did not persist on subsequent (two or more) cultures despite no treatment. For our centre, "cleared" is considered as the date treatment was stopped after 12 months of negative cultures (microbiological cure [13]), and "eradicated" as 12 months of negative cultures following stopping treatment. Approval for the study was granted through the hospital audit office (ref 288700). IBM SPSS Statistics (version 24) was used. Statistical significance was assumed at $\mathrm{p}<0.05$.

567 children with CF received care at RBH between 2011 and 2018. 63 new NTM isolates were found from 59 children (period prevalence 10.4\%); 34 MABSC (28 M. abscessus abscessus, six M. abscessus massiliense), $21 \mathrm{MAC}$, and eight other NTM species (four M. kansasii, three M. chelonae and one $M$. malmoense, all of which cleared without treatment). A median of six samples were taken per patient

@ERSpublications

Non-tuberculous mycobacteria pulmonary disease affects cystic fibrosis lung health and increasingly affects children. This study reports treatment outcomes from a large CF centre, with eradication rates exceeding $60 \%$ for $M$. abscessus complex infection. https://bit.ly/2L8TdFM

Cite this article as: Hughes DA, Bokobza I, Carr SB. Eradication success for non-tuberculous mycobacteria in children with cystic fibrosis. Eur Respir J 2021; 57: 2003636 [https://doi.org/10.1183/ 13993003.03636-2020]. 
during their treatment period. There was no difference in age, sex or body mass index z-score between groups, and all demonstrated highest prevalence in females (table 1). Median percent predicted forced expiratory volume in $1 \mathrm{~s}\left(\mathrm{ppFEV}_{1}\right)$ for those with $\mathrm{MAC}$ was lower in those who were treated, though this did not meet significance. All treated patients met ATS/Infectious Diseases Society of America criteria for NTM-PD [7].

For patients isolating MAC, 11 had transient infection (52\%), nine commenced treatment (43\%) and one did not start treatment. Four received intravenous (i.v.) initiation treatment with amikacin and meropenem. All treated patients received triple oral antibiotic maintenance therapy that included ethambutol and a macrolide. Six of the nine treated eradicated the infection (67\%), three of which had received i.v. initiation therapy. The most frequently seen co-infection within 1 year of starting treatment was Aspergillus fumigatus, found in seven (78\%).

Of those that grew MABSC, 26 started treatment (76\%), seven had transient infection (21\%) and one did not start treatment. All received i.v. initiation antibiotics, the majority for 3 weeks. All had triple antibiotic i.v. initiation regimes (all had amikacin, 24 meropenem with either cefoxitin or tigecycline) and a daily oral macrolide, regardless of erm-41 status. For the maintenance phase, all patients received nebulised amikacin in combination with dual or triple oral antibiotics including a macrolide. Of those treated for MABSC-PD, 21/26 (81\%) initially "cleared" the infection and $2 / 21(10 \%)$ of these regrew after stopping treatment. Overall, 17/26 (65\%) achieved eradication, 7/26 (27\%) failed to eradicate, and 2/26 (8\%) cleared the infection but transitioned before 12-month post-treatment follow-up. One of those who eradicated withdrew from full treatment after 4 months (poor drug tolerance). Eradication rates for M. abscessus massiliense were higher overall: 4/5 (80\%) eradicated with treatment and one spontaneously cleared. Receiving 2 weeks compared to 3 weeks of initiation treatment had no effect on eradication rates $(63 \%$ versus 69\%; nonsignificant). $\mathrm{ppFEV}_{1}$ at diagnosis did not predict eradication success (mean $81 \%$ pred versus $82 \%$ pred; nonsignificant). A functional erm-41 macrolide-resistance gene was identified in 10/16 (63\%) of M. abscessus abscessus first isolates that were tested and treated. Of the 13/16 that had completed treatment and 12-month post-treatment observation: 5/8 (63\%) eradicated if erm-41 active versus 4/5 (80\%) when erm-41 inactive. As with MAC patients, the most frequently occurring co-infection within 12 months of NTM isolation was Aspergillus fumigatus (54\%). Five of the MABSC group grew more than one species of NTM during the study period.

TABLE 1 Demographics and outcomes of M. abscessus complex (MABSC), M. avium complex (MAC) and other non-tuberculous mycobacteria (NTM) groups

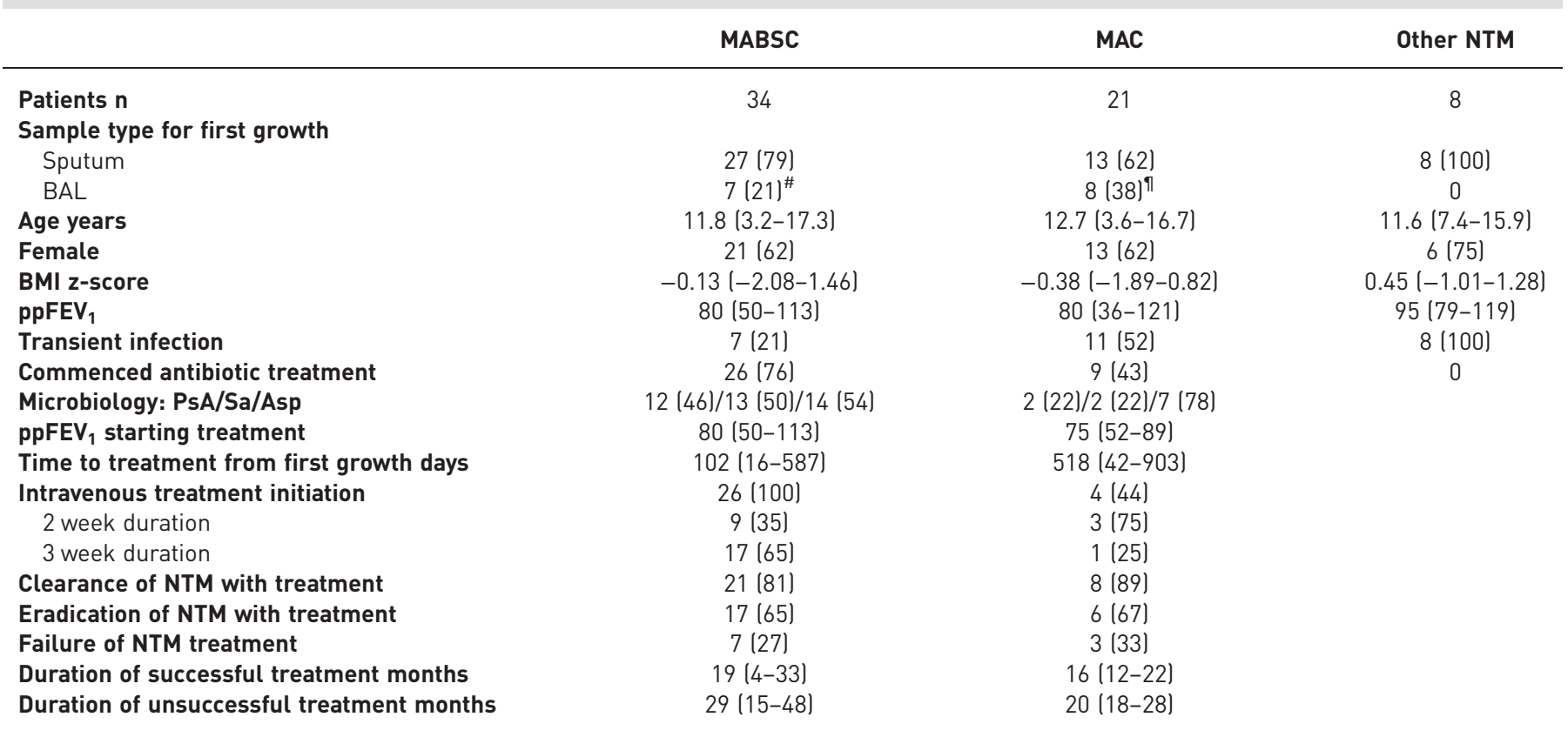

Data are presented as $\mathrm{n}(\%)$ or median (range), unless otherwise indicated. " : five had confirmation on subsequent sputum samples; the two patients diagnosed on bronchoalveolar lavage (BAL) alone had repeat BAL to look for clearance. ": four had confirmation on sputum and went on to treatment; two had repeat negative BAL samples, two negative subsequent sputum and were not treated. BMI: body mass index; $\mathrm{ppFEV}_{1}$ : percent of predicted forced expiratory volume in $1 \mathrm{~s}$; PsA: Pseudomonas aeruginosa; Sa: Staphylococcus aureus; Asp: Aspergillus sp. 
A sputum sample was successfully obtained in 23 (92\%) children at 6 months of treatment for MABSC-PD. In those going on to eradicate, $82 \%$ (14/17) had achieved culture conversion by then, compared to $29 \%(2 / 7)$ who failed to eradicate. Within our study period, we saw one regrowth 12 months after clearance that later cleared without treatment. $\mathrm{pFEV}_{1}$ recorded at the start and end of treatment (taken 20 months after starting treatment for those who failed eradication) showed a 7\% improvement for those who successfully eradicated or cleared MABSC, in contrast to a $3 \%$ decline in those who failed treatment.

This study from a large single centre aimed to establish NTM treatment outcomes in children with CF. This is especially pertinent in an era of increasing prevalence, with growing evidence of impact on outcomes and lung transplant eligibility [15].

The overall period prevalence of $10.4 \%$ is in line with current rates in the UK. Our data show higher transient infection rates for MAC and other NTM species compared to MABSC, which may reflect a less aggressive treatment start strategy for non-MABSC infections. This may also explain why patients treated for MAC-PD trend towards a lower $\mathrm{ppFEV}_{1}$ compared to those not treated. As expected, the duration of treatment is longer in those who fail to eradicate: the decision to cease treatment in patients with persistent positive cultures remains difficult.

Our data demonstrate an overall eradication rate of 65-73\% for MABSC-PD (depending on whether two patients lost to follow-up subsequently "eradicated"), and 78\% for MAC-PD. These rates are significantly higher than the $20-40 \%$ previously reported $[12,13]$, which may reflect lower levels of underlying lung disease in a paediatric population. This information will be valuable when discussing NTM treatment with patients and families. Early identification and treatment for MABSC in particular is thought to positively impact on eradication rates, placing additional emphasis on screening policies such as the use of induced sputum in non-productive patients [16].

These data have already affected practice within our centre; culture positivity after 6 months of treatment for MABSC-PD is seen as predicting treatment failure and a second course of i.v. antibiotics is given in the hope this will improve eradication rates. Our relatively small sample size makes it challenging to identify beneficial outcomes from eradication, though indications from $\mathrm{ppFEV}_{1}$ at the start and end of treatment suggest that eradication of MABSC is beneficial to lung health.

This is the first study to report NTM treatment outcomes specific to children with CF, and to suggest potential strategies for improving treatment success. A prospective randomised controlled trial is urgently needed.

Dominic A. Hughes ${ }^{1,2}$, Idan Bokobza ${ }^{2}$ and Siobhán B. Carr $\overbrace{}^{1,2}$

${ }^{1}$ National Heart and Lung Institute, Imperial College London, London, UK. ${ }^{2}$ Royal Brompton and Harefield NHS Foundation Trust, London, UK.

Correspondence: Dominic A. Hughes, National Heart and Lung Institute, Emmanuel Kaye Building, 1B Manresa Road, London, SW3 6LR, UK. E-mail: d.hughes17@imperial.ac.uk

Received: 25 Sept 2020 | Accepted: 28 Jan 2021

Acknowledgements: The authors thank Alice Davenport for support with data collection and the microbiology laboratory staff, in particular Peter Darby, at the Royal Brompton Hospital, London.

Conflict of interest: D.A. Hughes has nothing to disclose. I. Bokobza has nothing to disclose. S.B. Carr reports personal fees and other (advisory board work and lecture fees) from Chiesi Pharmaceuticals, non-financial support and other (advisory board work, lecture fees, travel support and steering committee work) from Vertex, other (advisory board work) from Zambon, other (consultancy fees) from Insmed, outside the submitted work.

\section{References}

1 Gardner AI, McClenaghan E, Saint G, et al. Epidemiology of nontuberculous mycobacteria infection in children and young people with cystic fibrosis: analysis of UK Cystic Fibrosis Registry. Clin Infect Dis 2019; 68: 731-737.

2 Hoefsloot W, van Ingen J, Andrejak C, et al. The geographic diversity of nontuberculous mycobacteria isolated from pulmonary samples: an NTM-NET collaborative study. Eur Respir J 2013; 42: 1604-1613.

3 Lee MR, Sheng WH, Hung CC, et al. Mycobacterium abscessus complex infections in humans. Emerg Infect Dis 2015; 21: 1638-1646.

4 Bryant JM, Grogono DM, Greaves D, et al. Whole-genome sequencing to identify transmission of Mycobacterium abscessus between patients with cystic fibrosis: a retrospective cohort study. Lancet 2013; 381: 1551-1560.

5 Harris KA, Underwood A, Kenna DT, et al. Whole-genome sequencing and epidemiological analysis do not provide evidence for cross-transmission of Mycobacterium abscessus in a cohort of pediatric cystic fibrosis patients. Clin Infect Dis 2015; 60: 1007-1016.

6 Qvist T, Taylor-Robinson D, Waldmann E, et al. Comparing the harmful effects of nontuberculous mycobacteria and Gram negative bacteria on lung function in patients with cystic fibrosis. J Cyst Fibros 2016; 15: 380-385. 
7 Griffith DE, Aksamit T, Brown-Elliott BA, et al. An official ATS/IDSA statement: diagnosis, treatment, and prevention of nontuberculous mycobacterial diseases. Am J Respir Crit Care Med 2007; 175: 367-416.

8 Floto RA, Olivier KN, Saiman L, et al. US Cystic Fibrosis Foundation and European Cystic Fibrosis Society consensus recommendations for the management of non-tuberculous mycobacteria in individuals with cystic fibrosis. Thorax 2016; 71: Suppl. 1, i1-i22.

9 Haworth CS, Banks J, Capstick T, et al. British Thoracic Society guidelines for the management of non-tuberculous mycobacterial pulmonary disease (NTM-PD). Thorax 2017; 72: Suppl. 2, iil-ii64.

10 Martiniano SL, Sontag MK, Daley CL, et al. Clinical significance of a first positive nontuberculous mycobacteria culture in cystic fibrosis. Ann Am Thorac Soc 2014; 11: 36-44.

11 van Ingen J, Aksamit T, Andrejak $\mathrm{C}$, et al. Treatment outcome definitions in nontuberculous mycobacterial pulmonary disease: an NTM-NET consensus statement. Eur Respir J 2018; 51: 1800170.

12 Qvist T, Gilljam M, Jonsson B, et al. Epidemiology of nontuberculous mycobacteria among patients with cystic fibrosis in Scandinavia. J Cyst Fibros 2015; 14: 46-52.

13 DaCosta A, Jordan CL, Giddings $\mathrm{O}$, et al. Outcomes associated with antibiotic regimens for treatment of Mycobacterium abscessus in cystic fibrosis patients. J Cyst Fibros 2017; 16: 483-487.

14 Royal Brompton Hospital paediatric CF team. Clinical Guidelines: Care of Children with Cystic Fibrosis, 2020. 8th Edn. London, Royal Brompton and Harefield NHS Foundation Trust, 2020. Available from: www.rbht.nhs.uk/ childrencf

15 Kavaliunaite E, Harris KA, Aurora P, et al. Outcome according to subspecies following lung transplantation in cystic fibrosis pediatric patients infected with Mycobacterium abscessus. Transpl Infect Dis 2020; 22: e13274.

16 Ahmed MI, Kulkarni H, Shajpal S, et al. Early detection of non-tuberculous mycobacteria in children with cystic fibrosis using induced sputum at annual review. Pediatr Pulmonol 2019; 54: 257-263. 